Annals of Pure and Applied Mathematics

Vol. 17, No. 1, 2018, 29-33

ISSN: 2279-087X (P), 2279-0888(online)

Published on 17 April 2018

www.researchmathsci.org

DOI: http://dx.doi.org/10.22457/apam.v17nla4

Annals of

Pure and Applied

Mathematics

\title{
Edge Co-PI Indices of Special Graphs
}

\section{G. Sharmila Devi}

Department of Mathematics, Kongu Arts and Science College (Autonomous)

Erode - 638107, Tamilnadu, India. E-mail: sharmilashamritha@gmail.com

Received 16 March 2018; accepted 16 April 2018

Abstract. The edge Co-PI index of a graph $G$, denoted by $C o-P I_{e}(G)$, is defined as $C o-P I_{e}(G)=\sum_{e=u v E(G)}\left|m_{u}^{G}(e)-m_{v}^{G}(e)\right|$, where $m_{u}(e)$ denotes the number of edges of $G$ whose distance to the vertex $u$ is less than the distance to the vertex $v$. In this paper, the upper bounds for the edge Co-PI indices of Corona product product of two connected graphs is obtained. Finally, we compute the edge Co-PI indices of Tetrameric 1, 3Adamantane.

Keywords: Co-PI index; corona graph

AMS Mathematics Subject Classification (2010): 05C12, $05 \mathrm{C} 76$

\section{Introduction}

All the graphs considered in this paper are connected and simple. A vertex $x \in V(G)$ is said to be equidistant from the edge $e=u v$ of $G$ if $d_{G}(u, x)=d_{G}(v, x)$, where $d_{G}$ $(u, x)$ denotes the distance between $u$ and $x$ in $G$. The degree of the vertex $u$ in $G$ is denoted by $d_{G}(u)$.

For an edge $u v=e \in E(G)$, the number of vertices of $G$ whose distance to the vertex $u$ is smaller than the distance to the vertex $v$ in $G$ is denoted by $n_{u}^{G}(e)$; analogously, $n^{G}{ }_{v}(e)$ is the number of vertices of $G$ whose distance to the vertex $v$ in $G$ is smaller than the distance to the vertex $u$; the vertices equidistant from both the ends of the edge $e=u v$ are not counted.

Similarly, $m_{u}(e)$ denotes the number of edges of $G$ whose distance to the vertex $u$ is less than the distance to the vertex $v$.

The vertex PI index of $\mathrm{G}$, denoted by $\mathrm{PI}(\mathrm{G})$, is defined as $P I(G)=\sum_{e=u v \in E(G)}\left(n_{u}^{G}(e)+n_{v}^{G}(e)\right)$. The

Co- PI index of G, denoted by Co - PI(G), is defined as Co-PI $(G)=\sum_{e=u v \in E(G)}\left|n_{u}^{G}(e)-n_{\nu}^{G}(e)\right|$.

The edge PI index of $\mathrm{G}$, denoted by $\mathrm{PI}_{\mathrm{e}}(\mathrm{G})$, is defined as $P I_{e}(G)=\sum_{e=u v E(G)}\left(m_{u}^{G}(e)+m_{v}^{G}(e)\right)$.

The edge Co-PI index of $\mathrm{G}$, denoted by Co-PI $(\mathrm{G})$, is defined as

$$
C o-P I_{e}(G)=\sum_{e=u v \in E(G)}\left|m_{u}^{G}(e)-m_{v}^{G}(e)\right| .
$$

The $P I$ index of the graph $G$ is a topological index related to equidistant vertices. Another topological index of $G$ related to distance of $G$ is the Wiener index of $G$, first introduced by Wiener, see [20]. Khadikar, Karmarkar and Agrawal [9] first introduced edge Padmakar-Ivan index of graphs and they investigated the chemical applications of the Padmakar-Ivan index. The mathematical properties of the $P I_{v}$ and 


\section{G. Sharmila Devi}

its applications in chemistry and nanoscience are well studied by Ashrafi and Loghman [2, 3], Ashrafi and Rezaei [4], Deng, Chen and Zhang [5], Khadikar [8], Klavzar [10] and Yousefi-Azari, Manoochehrian and Ashrafi [19]. The vertex PI indices of the tensor and strong products of graphs are studied in $[14,16]$. In $[11,18$, 12], the PI indices of bridge graphs and chain graphs are discussed. The properties of the edge Co-PI indices of graphs are discussed in [1]. In this paper, the upper bounds for the edge Co-PI indices of corona product and Tetrameric 1,3-Adamantane are obtained.

\section{Corona product}

Let $G$ and $H$ be two graphs. The corona product $G \circ H$, is obtained by taking one copy of $G$ and $|V(G)|$ copies of $H$; and by joining each vertex of the $i$-th copy of $H$ to the $i$-th vertex of $G$, where $1 \leq i \leq|V(G)|$, see Figure 1. For our convenience, we partition the edge set of $G \circ H$ into three sets, $E_{1}=\left\{e \in E(G \circ H) / e \in E\left(H_{i}\right), 1 \leq i \leq n\right\}$, $E_{2}=\{e \in E(G \circ H) / e \in E(G)\}$ and $E_{3}=\left\{e \in E(G \circ H) / e=u v, u \in V\left(H_{i}\right), 1 \leq i \leq n, v \in V(G)\right\}$.

It is easy to see that $\mathrm{E}_{1}, \mathrm{E}_{2}$ and $\mathrm{E}_{3}$ are partition of the edge set of $G \circ H$ and also $\left|E_{1}\right|=|V(G)||E(H)|,\left|E_{2}\right|=|E(G)|$ and $\left|E_{3}\right|=|V(G)||V(H)|$.

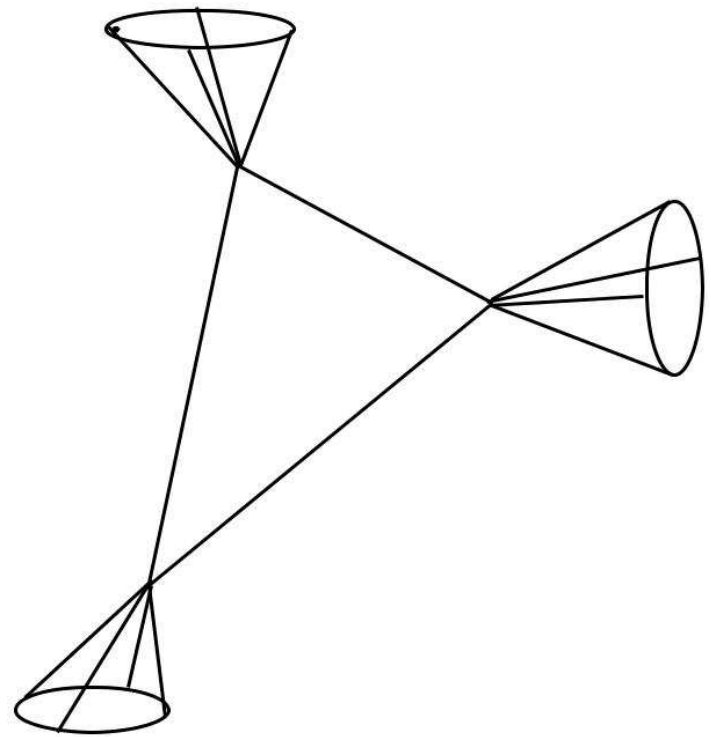

Figure 1: Corona product of $C_{3}$ and $C_{4}$

Theorem 2.1. Let $G$ be connected graph of order $n$ and size $p$. If $H$ is a triangle free and $r$ - regular graph of order $m$ and size $q$, then $\mathrm{Co}-\mathrm{PI}_{e}(G \circ H) \leq \mathrm{Co}-\mathrm{PI}_{e}(G)+$ $n\left(\mathrm{Co}-P I_{e}(H)\right)+(m+q) C o-P I(G)+n m(2 r-p-n(m+q)+1)$.

Proof: We partition the edges of $G \circ H$ into three sets $\mathrm{E}_{1}, \mathrm{E}_{2}$ and $\mathrm{E}_{3}$ defined above.

First we compute $\sum_{e=u v \in E_{1}}\left|m_{u}^{G \circ H}(e)-m_{v}^{G \circ H}(e)\right|$.

Let $e=u v \in E_{1}$.

Then from the structure of $G \circ H$, we have $m_{u}^{G \circ H}(e)=m_{u}^{G}(e)+(m+q) n_{u}^{G}(e)$ and $m_{v}^{G \circ H}(e)=m_{v}^{G}(e)+(m+q) n_{v}^{G}(e)$ 
Edge Co-PI Indices of Special Graphs

$$
\begin{aligned}
& \sum_{e=u v \in E_{1}}\left|m_{u}^{G \circ H}(e)-m_{v}^{G \circ H}(e)\right|=\sum_{e=u v \in E(G)}\left|\left(m_{u}^{G}(e)+(m+q) n_{u}^{G \circ H}(e)\right)\right| \\
= & \sum_{e=u v \in E(G)}\left|\left(\left(m_{u}^{G}(e)-m_{v}^{G}(e)\right)+(m+q)\left(n_{u}^{G \circ H}(e)\right)-n_{v}^{G \circ H}(e)\right)\right| \\
& \left.-m_{v}^{G}(e)+(m+q) n_{v}^{G \circ H}(e)\right) \\
\leq & \sum_{e=u v \in E(G)}\left|m_{u}^{G}(e)-m_{v}^{G}(e)\right|+(m+q) \sum_{e=u v \in E(G)}\left|n_{u}^{G}(e)-n_{v}^{G}(e)\right| . \\
= & \left(\mathrm{Co}-\mathrm{PI}_{\mathrm{e}}(\mathrm{G})+(\mathrm{m}+\mathrm{q})(\mathrm{Co}-\mathrm{PI}(\mathrm{G}) .\right.
\end{aligned}
$$

Next we compute $\sum_{e=u v \in E_{2}}\left|m_{u}^{G \circ H}(e)-m_{v}^{G \circ H}(e)\right|$.

Let $e=u v \in E_{2}$. Then from the structure of $G \circ H$, we have

$$
\begin{gathered}
m_{u}^{G \circ H}(e)=m_{u}^{H}(e)+1 \text { and } m_{v}^{G \circ H}(e)=m_{v}^{H}(e)+1 . \\
\sum_{e=u v \in E_{2}}\left|m_{u}^{G \circ H}(e)-m_{v}^{G \circ H}(e)\right|=\sum_{i=1}^{n} \sum_{e=u v \in E(G)}\left|\left(m_{u}^{H}(e)+1\right)-\left(m_{v}^{H}(e)+1\right)\right| \\
=n \sum_{e=u v \in E(H)}\left|m_{u}^{G}(e)-m_{v}^{G}(e)\right| . \\
=\mathrm{n}\left(\mathrm{Co}-\mathrm{PI}_{\mathrm{e}}(\mathrm{H})\right) .
\end{gathered}
$$

Finally, we compute $\sum_{e=u v \in E_{3}}\left|m_{u}^{G \circ H}(e)-m_{v}^{G \circ H}(e)\right|$.

Let $e=u v \in E_{3}$. Then from the structure of $G \circ H$, we have

$$
\begin{aligned}
& m_{u}^{G \circ H}(e)=d_{H}(u) \text { and } m_{v}^{G \circ H}(e)=|E(G \circ H)|-\left(d_{H}(u)+1\right) \\
& \sum_{e=u v \in E_{3}}\left|m_{u}^{G \circ H}(e)-m_{v}^{G \circ H}(e)\right|=\sum_{u \in V(H)} \sum_{v \in V(G)}\left|d_{H}(u)-\left(|E(G \circ H)|-\left(d_{H}(u)+1\right)\right)\right| \\
= & \sum_{u \in V(H)} \sum_{v \in V(G)}(r-p-n(m+q)+r+1) \\
\leq & n m(2 r-p-n(m+q)+1) .
\end{aligned}
$$

Now we shall obtain the $\mathrm{Co}-P I_{e}(G \circ H)$.

$$
\begin{aligned}
& \mathrm{Co}-\mathrm{PI}_{\mathrm{e}}(\mathrm{G} \circ \mathrm{H})=\sum_{e=u v \in E_{1}}\left|m_{u}^{G \circ H}(e)-m_{v}^{G \circ H}(e)\right|+\sum_{e=u v \in E_{2}}\left|m_{u}^{G \circ H}(e)-m_{v}^{G \circ H}(e)\right| \\
& +\sum_{e=u v \in E_{3}}\left|m_{u}^{G \circ H}(e)-m_{v}^{G \circ H}(e)\right| . \\
& \leq C o-P I_{e}(G)+n\left(C o-P I_{e}(H)\right)+(m+q) C o-P I(G)+n m(2 r-p-n(m+q)+1) .
\end{aligned}
$$

\section{Edge Co-PI index of Tetrameric 1,3-Adamantane}

From the structure of the graph tetrameric 1,3-adamantane $T A[n]$, the number of vertices and edges are $10 n$ and $13 n-1$, respectively, see Figure 2. 
G. Sharmila Devi

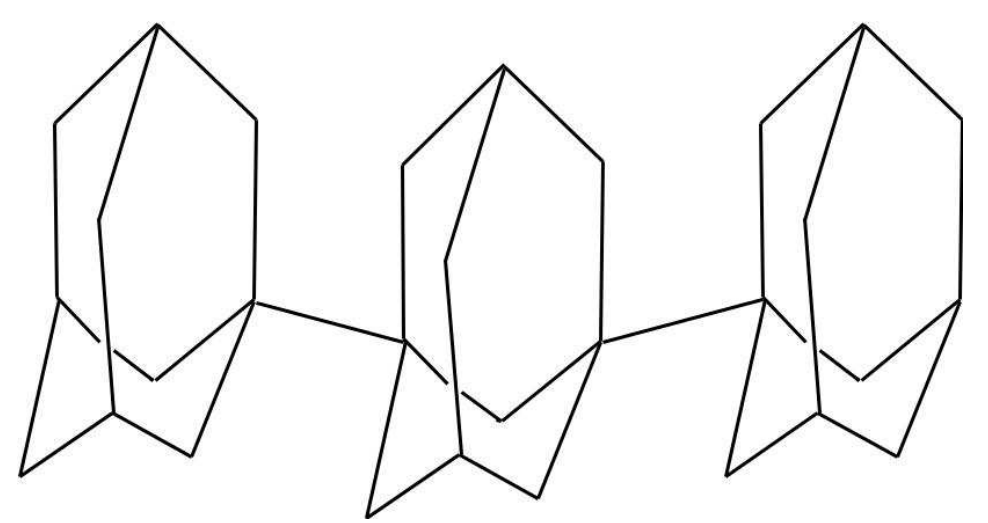

Figure 2: The tetrameric 1,3-adamantane (TA[3])

Theorem 3.1. The edge Co-PI index of $T A[n]$ is $C o-P I_{e}(T A[n]) \leq 18 n$.

Proof: From the structure of the graph $T A[n]$, we have the following cases of edges.

If $\mathrm{e}=\mathrm{u}_{\mathrm{i}} \mathrm{v}_{\mathrm{i}}$ then $m_{u_{i}}^{G}(e)=13 i-1$ and $m_{v_{i}}^{G}(e)=13(n-i)-1$.

If $\mathrm{e}=\mathrm{uv}=\left\{\mathrm{X}_{1} \mathrm{X}_{2}, \mathrm{X}_{5} \mathrm{u}_{\mathrm{k}}, \mathrm{X}_{6} \mathrm{X}_{7}\right\}$, then $m_{u}^{G}(e)=6+13(n-k)$ and $m_{v}^{G}(e)=3+13(k-1)$.

If $\mathrm{e}=\mathrm{uv}=\left\{\mathrm{X}_{1} \mathrm{X}_{4}, \mathrm{~V}_{\mathrm{k}-1} \mathrm{X}_{5}, \mathrm{X}_{7} \mathrm{X}_{8}\right\}$, then $m_{u}^{G}(e)=6+13(k-1)$ and $m_{v}^{G}(e)=3+13(n-k)$.

If $\mathrm{e}=\mathrm{uv}=\left\{\mathrm{X}_{2} \mathrm{~V}_{\mathrm{k}-1}, \mathrm{X}_{3} \mathrm{X}_{7}, \mathrm{X}_{4} \mathrm{U}_{\mathrm{k}}\right\}$, then $m_{u}^{G}(e)=3$ and $m_{v}^{G}(e)=6+13(n-k)+13(k-1)$.

If $\mathrm{e}=\mathrm{uv}=\left\{\mathrm{X}_{1} \mathrm{X}_{3}, \mathrm{u}_{\mathrm{k}} \mathrm{X}_{8}, \mathrm{~V}_{\mathrm{k}-1} \mathrm{X}_{6}\right\}$, then $m_{u}^{G}(e)=6+13(n-k)+13(k-1)$ and $m_{v}^{G}(e)=3$.

Hence, $C o-P I_{e}(G)=\sum_{e=u v \in E(G)}\left|m_{u}^{G}(e)-m_{v}^{G}(e)\right|$.

$=\sum_{i=1}^{n-1}\left|m_{u}^{G}(e)-m_{v}^{G}(e)\right|+3 \sum_{k=1}^{n}\left|m_{u}^{G}(e)-m_{v}^{G}(e)\right|+3 \sum_{i=1}^{n}\left|m_{u}^{G}(e)-m_{v}^{G}(e)\right|$

$+3 \sum_{k=1}^{n}\left|m_{u}^{G}(e)-m_{v}^{G}(e)\right|+3 \sum_{i=1}^{n}\left|m_{u}^{G}(e)-m_{v}^{G}(e)\right|$

$=\sum_{k=1}^{n-1}|(13 i-1)-(13(n-i)-1)|+3 \sum_{i=1}^{n}|(6+13(n-k))-(3+13(k-1))|$

$+3 \sum_{i=1}^{n}|(6+13(k-1))-(3+13(n-k))|$

$+3 \sum_{k=1}^{n}|3-(6+13(n-k)+13(k-1))|+3 \sum_{k=1}^{n}|6+13(n-k)+13(k-1)-3|$.

$\leq 18$ n.

\section{REFERENCES}

1. Arjomandfar and O.Khormali, On edge Co-PI indices, Int. J. Industrial Mathematics, 6 (2014) 59-64.

2. A.R.Ashrafi and A.Loghman, PI index of zig-zag polyhex nanotubes, MATCH Commun. Math. Comput. Chem., 55 (2006) 447-452.

3. A.R.Ashrafi and A. Loghman, PI index of armchair polyhex nanotubes, Ars Combin., 80 (2006) 193-199.

4. A.R.Ashrafi and F.Rezaei, PI index of polyhex nanotori, MATCH Commun. Math. Comput. Chem., 57 (2007) 243-250. 
Edge Co-PI Indices of Special Graphs

5. H.Deng, S.Chen and J.Zhang, The PI index of phenylenes, J. Math. Chem., 41 (2007) 63-69.

6. J.Devillers and A.T.Balaban, Eds., Topological indices and related descriptors in QSAR and QSPR, Gordon and Breach, Amsterdam, The Netherlands, 1999.

7. M.H.Khalifeh, H.Yousefi-Azari and A.R.Ashrafi, Vertex and edge PI indices of Cartesian product graphs, Discrete Appl. Math., 156 (2008) 1780-1789.

8. P.V.Khadikar, On a novel structural descriptor PI, Nat. Acad. Sci. Lett., 23 (2000) 113-118.

9. P.V.Khadikar, S.Karmarkar and V.K.Agrawal, A novel PI index and its Application to QSPR/QSAR studies, J. Chem. Inf. Comput. Sci., 41 (2001) 934-949.

10. S.Klavzar, On the PI index: PI-partitions and Cartesian product graphs, MATCH Commun. Math. Comput. Chem., 57 (2007) 573-586.

11. T.Mansour and M.Schork, The PI index of bridge and chain graphs, MATCH Communications in Mathematical and in Computer Chemistry, 61(3) (2009) 723734.

12. T.Mansour and M.Schork, The vertex PI index and Szeged index of bridge graphs, Discrete Applied Mathematics, 157(7) (2009) 1600-1606.

13. T.Mansour and M.Schork, The PI index of polyomino chains of $4 \mathrm{k}-\mathrm{cycles}$, Acta Applicandae Mathematicae, 109 (2010) 671-681.

14. K.Pattabiraman and P.Paulraja, On some topological indices of the tensor products of graphs, Discrete Appl. Math., 160 (2012) 267-279.

15. K.Pattabiraman and P.Paulraja, Vertex and edge Padmakar-Ivan indices of generalized hierarchical product of graphs, Discrete Appl. Math., 160 (2012) 13761384.

16. K.Pattabiraman and P.Paulraja, Wiener and vertex PI indices of the strong product of graphs, Discuss. Math. Graph Theory, 32 (2012) 749-769.

17. G.Sharmila Devi and V.Kaladevi, Co-PI index of some chemical graphs, Gen. Math. Notes, 34 (2016) 64-77.

18. G.Sharmila Devi and V.Kaladevi, Vertex and edge Co-PI indices of bridge graph, Int. Journal of Advanced Mathematical Sciences, 6 (2018) 10-12.

19. H.Yousefi-Azari, B.Manoochehrian and A.R.Ashrafi, The PI index of product graphs, Appl. Math. Lett., 21 (2008) 624-627.

20. H.Wiener, Structural determination of the paraffin boiling points, J. Am. Chem. Soc., 69 (1947) 17-20. 